\title{
4D Flow and 2D PC MRI: impact of volumetric coverage and three-directional velocity encoding on quantification of aortic hemodynamics
}

\author{
Emilie Bollache ${ }^{1 *}$, Pim van Ooij ${ }^{1}$, Alexander L Powell ${ }^{1}$, James C Carr ${ }^{1}$, Michael Markl ${ }^{1,2}$, Alex J Barker ${ }^{1}$ \\ From 19th Annual SCMR Scientific Sessions \\ Los Angeles, CA, USA. 27-30 January 2016
}

\section{Background}

The accurate assessment of arterial blood flow is crucial for the diagnosis and management of patients with cardiovascular disease. Our aim was to systematically compare three phase-contrast (PC) MRI sequences for the quantification of aortic hemodynamics, including: 1) 2D time-resolved (CINE) PC MRI with one-directional through-plane (2D-1dir) velocity encoding; 2) 2D CINE PC MRI with three-directional (2D-3dir) velocity encoding; 3) 4D flow MRI with full volumetric coverage of the aorta and three-directional velocity encoding.

\section{Methods}

We studied 15 healthy volunteers (51 \pm 19 yrs; 11 men) with MRI (1.5T MAGNETOM Aera, Siemens). 4D flow MRI was acquired in a sagittal oblique $3 \mathrm{D}$ volume covering the thoracic aorta (free breathing; spatial resolution $(\mathrm{sr})=3.1 \times 2.3 \times 2.5 \mathrm{~mm}^{3}$; temporal resolution $(\operatorname{tr})=39.2 \mathrm{~ms})$. In addition, 2D-1dir (breath-hold; $\mathrm{sr}=$ $1.8 \times 2.5 \times 6 \mathrm{~mm}^{3} ; \mathrm{tr}=38.4 \mathrm{~ms}$ ) and $2 \mathrm{D}$-3dir (free breathing; $\mathrm{sr}=1.8 \times 2.5 \times 6 \mathrm{~mm}^{3} ; \mathrm{tr}=38.4 \mathrm{~ms}$ ) CINE PC MRI were acquired in a $2 \mathrm{D}$ plane perpendicular to both the ascending (AA) and descending (DA) aorta. For all acquisitions, the velocity sensitivity (venc) was $150 \mathrm{~cm} / \mathrm{s}$ and prospective ECG gating was used. All 2D CINE PC and 4D flow MRI data were preprocessed to correct for Maxwell terms, velocity aliasing and eddy currents (Figure 1). 4D flow MRI data analysis included the 3D segmentation of the aorta (Mimics, Materialise) with a plane placed orthogonal to the aortic centerline (Ensight, CEI) and coregistered to the scanner coordinates of the 2D slices used for the 2D CINE PC MRI

'Department of Radiology, Northwestern University, Chicago, IL, USA Full list of author information is available at the end of the article acquisitions. Delineation of the AA and DA vessel lumen contours enabled the measurement of net flow volume $\left(\mathrm{Q}_{\text {net }}\right)$, peak flow $\left(\mathrm{Q}_{\max }\right)$ and peak velocity at both locations. Peak velocity was calculated using the through-plane component only $\left(\mathrm{Vz}_{\max }\right)$ for the 3 sequences, or the Euclidian norm of the three principal directions $\left(\right.$ Vmag $\left._{\max }\right)$ for $4 \mathrm{D}$ flow and 2D-3dir CINE PC MRI.

\section{Results}

All 2D-1dir CINE PC MRI indices in both the AA and DA were significantly lower than 2D-3dir indices, while a better agreement was found between $4 \mathrm{D}$ flow and 2D3 dir measurements (Figure 2). Differences between 2D1 dir and 2D-3dir indices, in percentage of the mean value, were lower in the DA than in the AA (Table), suggesting that flow is more complex in the AA and three-directional encoding is required to fully capture flow dynamics.

\section{Conclusions}

Our results suggest the importance of three-directional encoding of velocity for estimation of hemodynamic indices, especially when considering volume and peak velocity measured in the AA. Differences between the clinically used 2D-1dir and 2D-3dir CINE PC MRI sequences could also be attributed to changes in aortic hemodynamics between breath-hold and free-breathing acquisitions, highlighting the importance of technique-specific reference values for aortic flow and velocity indices.

\footnotetext{
Authors' details

'Department of Radiology, Northwestern University, Chicago, IL, USA.

'Department of Biomedical Engineering, Northwestern University, Chicago, IL, USA.
} 

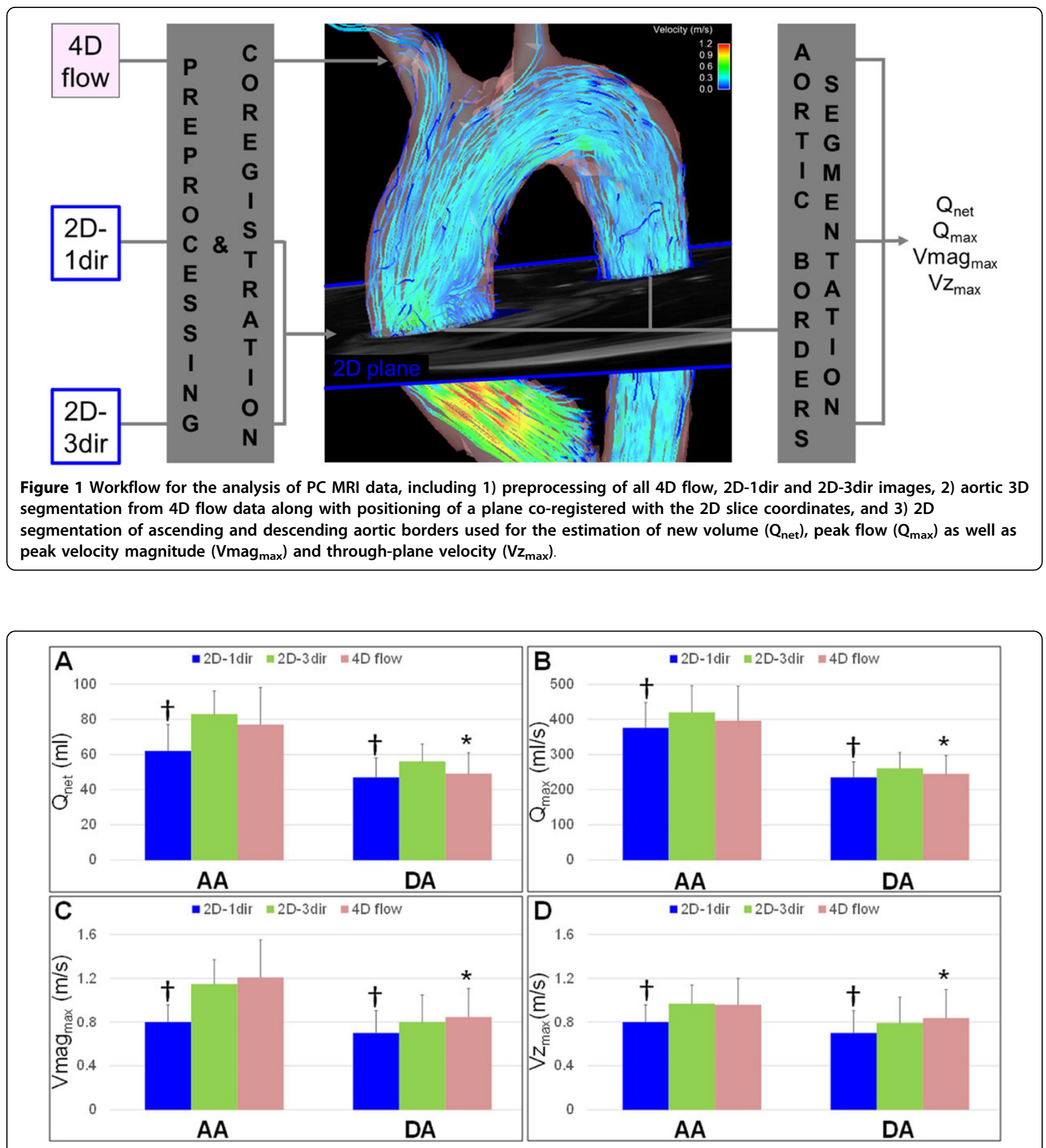

Figure 2 